\title{
Belinostat, at Its Clinically Relevant Concentrations, Inhibits Rifampicin-Induced CYP3A4 and MDR1 Gene Expression $\$$
}

\author{
Kodye L. Abbott, Chloe S. Chaudhury, Aneesh Chandran, Saraswathi Vishveshwara, \\ Zdenek Dvorak, Eva Jiskrova, Karolina Poulikova, Barbora Vyhlidalova, Sridhar Mani, \\ and Satyanarayana R. Pondugula
}

\begin{abstract}
Department of Anatomy, Physiology and Pharmacology (K.L.A., C.S.C., S.R.P.) and Auburn University Research Initiative in Cancer (K.L.A., C.S.C., S.R.P.), Auburn University, Auburn, Alabama; Molecular Biophysics Unit, Indian Institute of Science, Bangalore, India (A.C., S.V.); Regional Centre of Advanced Technologies and Materials, Faculty of Science, Palacky University, Olomouc, Czech Republic (Z.D., E.J., K.P., B.V.); and Albert Einstein Cancer Center, Albert Einstein College of Medicine, New York, New York (S.M.)
\end{abstract}

Received September 18, 2018; accepted January 3, 2019

\section{ABSTRACT}

Activation of human pregnane $X$ receptor (hPXR) has been associated with induction of chemoresistance. It has been proposed that such chemoresistance via cytochrome P450/drug transporters can be reversed with the use of antagonists that specifically abrogate agonist-mediated hPXR activation. Unfortunately, proposed antagonists lack the specificity and appropriate pharmacological characteristics that allow these features to be active in the clinic. We propose that, ideally, an hPXR antagonist would be a cancer drug itself that is part of a "cancer drug cocktail" and effective as an hPXR antagonist at therapeutic concentrations. Belinostat (BEL), a histone deacetylase inhibitor approved for the treatment of relapsed/refractory peripheral T-cell lymphoma, and often used in combination with chemotherapy, is an attractive candidate based on its hPXR ligandlike features. We sought to determine whether these features of BEL might allow it to behave as an antagonist in combination

\section{Introduction}

The human pregnane $\mathrm{X}$ receptor (hPXR) is the master regulator of CYP3A4 and MDR1 (ABCB1 or P-glycoprotein) gene expression (Chen et al., 2012). CYP3A4 and MDR1 together

This work was supported by the Auburn University Research Initiative in Cancer Grant (S.R.P.) and an Animal Health and Disease Research Grant (S.R.P.). The work was also supported by the National Cancer Institute (NCI)/ National Institutes of Health (NIH)/Department of Health and Human Services (HHS) [Grants P30-CA-013330/CA/NCI NIH HHS/United States (Principal Investigator, D. Goldman), R01-CA-161879/CA/NCI NIH HHS/United States (S.M.), and R01-CA-222469/NCI/NIH (S.M.)]; the Broad Medical Research Program [Grant 362520] (S.M.); the Department of Defense [Grant PR-160167] (S.M.); the Operational Programme Research, Development and EducationEuropean Regional Development Fund; and the Ministry of Education, Youth and Sports of the Czech Republic [Grant CZ.02.1.01/0.0/0.0/16_019/ 0000754] (Z.D.). S.V. and A.C. are supported by the National Academy of Sciences, India.

Part of this work was presented as follows: Abbott KL, Chaudhury CS, Narayanan N, and Pondugula SR (2018) Belinostat inhibits rifampicin induced CYP3A4 and MDR1 gene expression. Experimental Biology 2018; 2018 April 21-25; San Diego, CA. American Association of Anatomists, Rockville, MD; The American Physiological Society, Rockville, MD; American Society for Biochemistry and Molecular Biology, Rockville, MD; American Society for Investigative Pathology, Rockville, MD; American Society for Pharmacology and Experimental Therapeutics, Rockville, MD.

https://doi.org/10.1124/mol.118.114587.

S This article has supplemental material available at molpharm. aspetjournals.org. chemotherapy regimens that include hPXR activators. BEL represses agonist-activated hPXR target gene expression at its therapeutic concentrations in human primary hepatocytes and LS174T human colon cancer cells. BEL repressed rifampicininduced gene expression of CYP3A4 and multidrug resistance protein 1 , as well as their respective protein activities. BEL decreased rifampicin-induced resistance to $\mathrm{SN}-38$, the active metabolite of irinotecan, in LS174T cells. This finding indicates that BEL could suppress hPXR agonist-induced chemoresistance. BEL attenuated the agonist-induced steroid receptor coactivator-1 interaction with hPXR, and, together with molecular docking studies, the study suggests that BEL directly interacts with multiple sites on hPXR. Taken together, our results suggest that BEL, at its clinically relevant therapeutic concentration, can antagonize hPXR agonist-induced gene expression and chemoresistance.

contribute to the metabolism and disposition of more than $50 \%$ of clinically active drugs (Veith et al., 2009). Therefore, during multidrug chemotherapy, drug induction of hPXRmediated CYP3A4 and MDR1, can affect the therapeutic response of coadministered drugs, leading to chemoresistance. For instance, coadministration of an hPXR agonist resulted in the potential loss of efficacy of several chemotherapy drugs, such as irinotecan (Hu et al., 2007), imatinib (Borrelli and Izzo, 2009), cabozantinib (Nguyen et al., 2015), cobimetinib (Choo et al., 2015), and vemurafenib (MacLeod et al., 2015).

To overcome the hPXR agonist-induced chemoresistance, it is possible to inhibit the drug-activated hPXR with an hPXR antagonist. In this regard, some compounds and extracts have been reported to antagonize the agonist-activated hPXR target gene expression (Mani et al., 2013; Chai et al., 2016). The first hPXR antagonist reported was ecteinascidin-743, which was subsequently followed by reports that included plant extracts or compounds, such as ketoconazole (KET), fluconazole, enilconazole, sulforaphane, coumestrol, A792611 [(S)-1-[(1S,3S,4S)-4-[(S)-2-(3-benzyl-2-oxo-imidazolidin-1-yl)3,3-dimethyl-butyrylamino]-3-hydroxy-5-phenyl-1-(4-pyridin2-yl-benzyl)-pentylcarbamoyl]-2,2-dimethyl-propyl-carbamic 
acid methyl ester], camptothecin, FLB-12 [1-(4-(4-(((2R,4S)-2(2,4-difluorophenyl)-2-methyl-1,3-dioxolan-4-yl)methoxy)phenyl) piperazin-1-yl)ethanone], metformin, sesamin, fucoxanthin, milk thistle, bitter melon, and allyl isothiocyanate. However, these compounds and extracts fall short of their clinical utility, as it is unlikely for them to achieve in vivo concentration levels to antagonize hPXR without causing severe unacceptable toxicity. We therefore investigated clinically used drugs with this property, in that these compounds could serve to inhibit hPXR at therapeutically achieved concentrations.

Belinostat (BEL) is a small-molecule inhibitor of histone deacetylases that was approved by the Food and Drug Administration in 2014 for treating peripheral T-cell lymphoma. BEL is commonly being administered with combination chemotherapy protocols in the lymphoma [e.g., CHOP (cyclophosphamide, hydroxydaunorubicin [doxorubicin], oncovin [vincristine], prednisone) protocol] (Laribi et al., 2018). Steroids are also mainstay drugs in such protocols for this condition, and they are known to strongly elicit hPXR activity (El-Sankary et al., 2000; Pascussi et al., 2000, 2001; Wang et al., 2003; Dvorak and Pavek, 2010; Banerjee et al., 2013) and can contribute to chemoresistance (Lin and Wang, 2016). BEL was also being evaluated in human clinical trials for repurposing its use in treating other chemoresistant cancers. For example, recently completed and ongoing clinical trials in human lung cancer patients [clinical trial registry numbers NCT00926640 and NCT01090830 (clinicaltrials.gov)] show the use of BEL in combination chemotherapy with hPXR activators such as cisplatin (Masuyama et al., 2005), etoposide (Schuetz et al., 2002; Chang and Waxman, 2006), and paclitaxel (Synold et al., 2001; Masuyama et al., 2005; Chang and Waxman, 2006). Thus, if BEL, which has hPXR ligand features in silico (Xiao et al., 2011), could serve to antagonize the agonist-elicited hPXR activity, then it is possible that chemoresistance could be alleviated in some cases. However, it is unknown whether BEL antagonizes drug-activated hPXR target gene expression. In the current study, we show that BEL, at its clinical therapeutic concentration, can antagonize agonist-activated hPXR and chemoresistance.

\section{Materials and Methods}

Chemicals. DMSO ( $\geq 99.9 \%)$, rifampicin (RIF; $\geq 97 \%)$, SR 12813 [tetraethyl 2-(3,5-di-tert-butyl-4-hydroxyphenyl)ethenyl-1,1-bisphosphonate] (SR; $\geq 98 \%$ ), pregnenolone $16 \alpha$-carbonitrile $(\mathrm{PCN} ; \geq 97 \%)$, Ketoconazole (KET; $\geq 98 \%$ ), valspodar [PSC-833 (3S,6S,9S,12R,15S,18S,21S,24S,30S,33S)6,9,18,24-tetraisobutyl-3,21,30-triisopropyl-1,4,7,10,12,15,19,25,28nonamethyl-33-[(2R,4E)-2-methyl-4-hexenoyl]-1,4,7,10,13,16,19,22,25,28, 31-undecaazacyclotritriacontane-2,5,8,11,14,17,20,23,26,29,32-undecone); $\geq 98 \%$, and rhodamine 123 (R123; $\geq 85 \%$ ) were purchased from Sigma-Aldrich (St. Louis, MO). Belinostat (BEL; $\geq 99.74 \%$ ) was purchased from Selleck Chemicals (Houston, TX).

Cell Culture. LS174T human colon cancer cells were obtained from the American Type Culture Collection and grown in Dulbecco's modified Eagle's medium (HyClone Laboratories, Inc., Logan, UT) supplemented with 10\% FBS and the other additives (HyClone Laboratories, Inc.), as described previously (Pondugula et al., 2015b). The assay media included phenol red-free Dulbecco's modified Eagle's medium supplemented with 5\% charcoal/dextran-treated FBS (HyClone Laboratories, Inc.) and the other additives. Cryopreserved human
TABLE 1

Identification number, sex, race, and age of the hepatocyte donors

\begin{tabular}{llll}
\hline \multicolumn{1}{c}{ Identification Number } & Sex & \multicolumn{1}{c}{ Race } & $\begin{array}{c}\text { Age } \\
\text { (years) }\end{array}$ \\
\hline Thermo Fisher Scientific & & & \\
Hu8210 & Male & Caucasian & 51 \\
Hu8164 & Male & Caucasian & 23 \\
Triangle Research Laboratories & & & \\
HUM4111A & Male & Caucasian & 29 \\
HUM4113 & Male & Caucasian & 29 \\
HUM4119E & Female & African American & 30 \\
HUM4122B & Male & Asian & 35 \\
\hline
\end{tabular}

primary hepatocytes and hepatocyte media were purchased from Thermo Fisher Scientific (Waltham, MA) and Triangle Research Labs (Research Triangle, NC). The donor information for the hepatocytes is provided in Table 1 . The hepatocytes were cultured by following the manufacturer instructions and our published protocol (Pondugula et al., 2015b). Rat primary hepatocytes were isolated and cultured as described in the Supplemental Material. LS174T cells and the hepatocytes were treated with the vehicle or drugs for 24 hours before harvesting the cells for gene expression and/or functional studies.

Cell Viability Assays. Cell viability was measured using the MTT assays or CellTiter-Glo Luminescent Cell Viability Assays (Promega, Madison, WI) (Pondugula et al., 2015a,b,c).

RNA Isolation and Quantitative Reverse-Transcription Polymerase Chain Reaction Analysis. Total RNA was extracted from human primary hepatocytes, rat primary hepatocytes, and LS174T cells using the E.Z.N.A. Total RNA Kit I (Omega Bio-Tek, Norcross, GA). The quality and quantity of the total RNA was assessed using a NanoVuePlus Spectrophotometer (GE Healthcare, Chicago, IL). Reverse transcription was performed with the iScript cDNA Synthesis Kit (Bio-Rad, Hercules, CA). Quantitative polymerase chain reaction was performed by using the PerfeCTa SYBR Green FastMix (Quanta BioSciences, Beverly, MA) and CFX96 Touch Real-Time PCR Detection System (Bio-Rad). Transcripts of the housekeeping gene (18S ribosomal RNA or glyceraldehyde-3-phosphate dehydrogenase), CYP3A4, MDR1, and Cyp3a1 were amplified using the gene-specific primers (Supplemental Table 1). The comparative $C_{t}$ method was used for relative quantification for gene expression.

CYP3A4 Activity Assays. CYP3A4 activity was measured in the human primary hepatocytes using the luminescent P450-Glo CYP3A4 Cell-Based Assays (Promega) (Lee et al., 2010; Takeshita et al., 2011). The cultured hepatocytes were treated with DMSO or compounds for 24 hours, and the culture media were aspirated, washed with the maintenance medium (to determine the transcriptional effects of the compounds), and incubated with the luminogenic substrate (LuciferinIPA) in the culture medium for 45 minutes at $37^{\circ} \mathrm{C}$. The medium was subsequently transferred to 96 -well opaque white plates (PerkinElmer, Waltham, MA), and an equal volume of Luciferin Detection Reagent (Promega) was added to initiate a luminescent reaction. The plates were incubated at room temperature for 20 minutes before measuring the luminescence using a FLUOstar Optima Plate Reader (BMG Labtech, Cary, NC). KET was used as an inhibitor of hPXR as well as CYP3A4. The CellTiter-Glo Luminescent Cell Viability Assays were performed on the hepatocytes, after collecting the medium for the enzymatic activity, to normalize CYP3A4 activity to the cell number.

Intracellular R123 Accumulation Assays. The efflux activity of MDR1 was determined by measuring the intracellular accumulation of the fluorescent probe R123, as described previously (Pondugula et al., 2015a,b). In brief, LS174T cells were treated with DMSO or compounds for 24 hours in the assay media at $37^{\circ} \mathrm{C}$. The cells were

ABBREVIATIONS: BEL, belinostat; CYP3A4, cytochrome p450 3A4; GA, genetic algorithm; GST, glutathione S-transferase; hPXR, human pregnane $X$ receptor; KET, ketoconazole; LBD, ligand-binding domain; LBP, ligand-binding pocket; MDR1, multidrug resistance protein 1; PCN, pregnenolone 16 $\alpha$-carbonitrile; PSC-833, valspodar; PXR, pregnane X receptor; R123, rhodamine 123; RIF, rifampicin; rPXR, rat pregnane X receptor; SR, SR12813; SRC-1, steroid receptor coactivator-1; $t_{1 / 2}$, half-life; TR-FRET, time-resolved fluorescence energy transfer. 
then washed with PBS and incubated at $37^{\circ} \mathrm{C}$ for 15 minutes with or without the MDR1-specific inhibitor PSC-833 $(10 \mu \mathrm{M})$ in the assay media. Then, R123 ( $5 \mu \mathrm{M})$ was added to the cells in the presence or absence of PSC-833 and incubated for another 45 minutes. The media were aspirated, and the cells were washed with ice-cold PBS and lysed with distilled water. To determine the intracellular concentration of R123, the fluorescence was measured using an Infinite Microplate Reader (TECAN, Männedorf, Switzerland) at an excitation wavelength of $485 \mathrm{~nm}$ and an emission wavelength of $538 \mathrm{~nm}$.

Steroid Receptor Coactivator-1 Coactivator Recruitment Assays. LanthaScreen TR-FRET (time-resolved fluorescence energy transfer) Assays (Thermo Fisher Scientific) were performed to determine the steroid receptor coactivator-1 (SRC-1) interaction with hPXR according to the manufacturer instructions (Thermo Fisher Scientific). The assays were carried out in 384-well solid black plates. Briefly, a mixture of $10 \mathrm{nM}$ purified glutathione $S$-transferase (GST)-hPXR ligand-binding domain (LBD), $10 \mathrm{nM}$ terbium-labeled anti-GST antibody, and 500 nM Fl-SRC1-4 peptide was added to each well containing DMSO, SR (a known hPXR agonist), BEL (test compound) with or without SR, or KET (a known PXR inhibitor) with or without SR at different concentrations. The plates were incubated at room temperature in the dark for 1 hour. Terbium-labeled antibody was excited at $340 \mathrm{~nm}$, and emission intensities from terbium and fluorescein were detected at 490 and $520 \mathrm{~nm}$, respectively. A postexcitation lag time of 100 microseconds followed by an integration time of 200 microseconds was used to collect the time-resolved emission signals on a SpectraMax iD5 Microplate Reader (Molecular Devices, San Jose, CA) to calculate the 520/490 TR-FRET ratio.

hPXR Ligand-Binding Assays. The hPXR ligand-binding assays of BEL were performed using the LanthaScreen TR-FRET hPXR Competitive Binding Assay Kit (Thermo Fisher Scientific) according to the manufacturer instructions. The assays were performed in a volume of $20 \mu \mathrm{l}$ in 384-well black plates containing different concentrations of tested compounds in the range of $1 \mathrm{nM}$ to $100 \mu \mathrm{M}$, DMSO and SR $(100 \mu \mathrm{M})$ were used as a negative and a positive control, respectively. The reaction mixture was incubated at room temperature for 1 hour in dark, and fluorescent signals were then measured at 495 and $520 \mathrm{~nm}$, applying the excitation filter $340 \mathrm{~nm}$, on an Infinite F200 Microplate Reader (TECAN). Finally, the TR-FRET ratio was calculated by dividing the emission signal of $520 \mathrm{~nm}$ by that at $495 \mathrm{~nm}$. $\mathrm{hPXR}$-binding assays were performed in three independent experiments. The final $\mathrm{IC}_{50}$ value was obtained by processing the data with GraphPad Prism version 8.0 (GraphPad Software, San Diego, CA) using standard curve interpolation [sigmoidal, 4PL (fourth party logistics), and variable slope].

Human PXR Molecular Docking Studies. Ensemble-based molecular docking was performed using GOLD suite version 5.5.0 (Cambridge Crystallographic Data Centre, Cambridge, UK) (Jones et al., 1997). GOLD uses a genetic algorithm (GA) to explore the conformational flexibility of the ligand and receptor side chains in the binding pocket. Thirty centroid conformations of apo hPXR generated using a root-mean-square deviation-based clustering algorithm, obtained from previous work, were used for the docking (Chandran and Vishveshwara, 2016). In all the protein conformations, water and ions were removed prior to docking. For the docking purpose, a binding site was defined by considering all atoms within $12 \AA$ from the geometrical center of the docking site. For each of the 30 independent GA runs, a maximum number of 200 GA operations were performed. The docked complexes were ranked with GoldScore and then rescored using a ChemScore fitness function (Jones et al., 1997). BEL and KET were docked at multiple sites, whereas SR and RIF were docked at ligand-binding pocket (LBP) as controls. The scoring functions account for the hydrogen bonding, Van der Waals interactions, and steric complementarity between the ligand and receptor. For each ligand, the best ranked docked pose with corresponding ChemScore is considered for further analysis. The methodology used for the rodent PXR molecular docking studies is described in the Supplemental Material.
hPXR Reporter Gene Assays. The methodology used for the hPXR reporter gene studies is described in the Supplemental Material.

Data and Statistical Analysis. Data are shown as the mean \pm S.D. Analyses were performed using GraphPad Prism version 8.0 software. The significance of the differences between groups was evaluated by analysis of variance (ANOVA), followed by Dunnett's multiple-comparisons test. $P<0.05$ values were determined to be statistically significant.

\section{Results}

BEL, at Its Therapeutically Relevant Concentrations, Inhibits Rifampicin-Induced CYP3A4 and MDR1 Gene Expression. To identify clinical anticancer drugs for their ability to modulate hPXR target gene expression at their clinically relevant concentrations, we sought a small-scale cell-based screening approach using human hepatocells (Zuo et al., 2017). We identified BEL as one of the drugs that inhibits RIF-induced CYP3A4 gene expression (Supplemental Fig. 1) $(P<0.05)$. BEL is also an attractive candidate based on its hPXR ligand-like features in silico (Xiao et al., 2011). These observations led us to hypothesize that BEL, at its therapeutic concentrations, antagonizes agonist-induced hPXR target gene expression.

In humans, after a single 30-minute intravenous infusion of BEL at the recommended dosage of $1000 \mathrm{mg} / \mathrm{m}^{2}$ (Food and Drug Administration, 2014; Poole, 2014), the $C_{\max }$ of BEL could reach to $\sim 128 \pm 44 \mu \mathrm{M}$ (Yeo et al., 2012; Piper et al., 2014; Calvo et al., 2016). The time to plasma $C_{\max }$ can be reached in about 30 minutes after the start of infusion (Yeo et al., 2012; Piper et al., 2014; Calvo et al., 2016). BEL is rapidly metabolized, with a plasma half-life $\left(t_{1 / 2}\right)$ of $2-4$ hours (Yeo et al., 2012; Piper et al., 2014; Bailey et al., 2016; Calvo et al., 2016). The plasma pharmacokinetic parameters from the previous studies in humans are presented in Table 2 . The treatment regimen for the lymphoma includes repeat dosing of BEL at $1000 \mathrm{mg} / \mathrm{m}^{2}$ once daily on days $1-5$ of 21 -day cycles. BEL is not expected accumulate after the recommended repeat dosing (Yeo et al., 2012; Bailey et al., 2016; Calvo et al., 2016). In vitro plasma protein binding studies showed that approximately $95 \%(92.9 \%-95.8 \%)$ of BEL is bound to protein in an equilibrium dialysis assay (Food and Drug Administration, 2014; Poole, 2014). Based on these studies, the extrapolated therapeutic free plasma concentration of BEL during chemotherapy would be equivalent to $\sim 6 \mu \mathrm{M}$ with reference to the mean $C_{\max }$ and $\sim 3 \mu \mathrm{M}$ with reference to $50 \%$ mean $C_{\max }$ at the $t_{1 / 2}$ (2-4 hours). Based on the excretion studies in animals, it was estimated that BEL would be excreted to levels below expected pharmacologic activity by $\sim 5$ hours (Calvo et al., 2016). We therefore examined the effect of BEL, at its therapeutic free plasma concentrations, on agonist-induced endogenous hPXR target gene expression in human primary hepatocytes and LS174T human intestinal cells.

KET, a known antagonist of PXR (Huang et al., 2007), inhibited the hPXR agonist RIF-induced CYP3A4 mRNA expression in human primary hepatocytes (Fig. 1A), and CYP3A4 (Fig. 1C) and MDR1 (Fig. 1D) mRNA expression in LS174T colon cancer cells $(P<0.05)$. Similarly, BEL repressed RIF-induced CYP3A4 (Fig. 1, A and C) and MDR1 (Fig. 1D) mRNA levels $(P<0.05)$. We also determined whether BEL inhibits another hPXR agonist SR-induced gene expression. Indeed, BEL repressed SR-induced CYP3A4 gene 
TABLE 2

Published plasma pharmacokinetic parameters of BEL in humans after a single intravenous dose

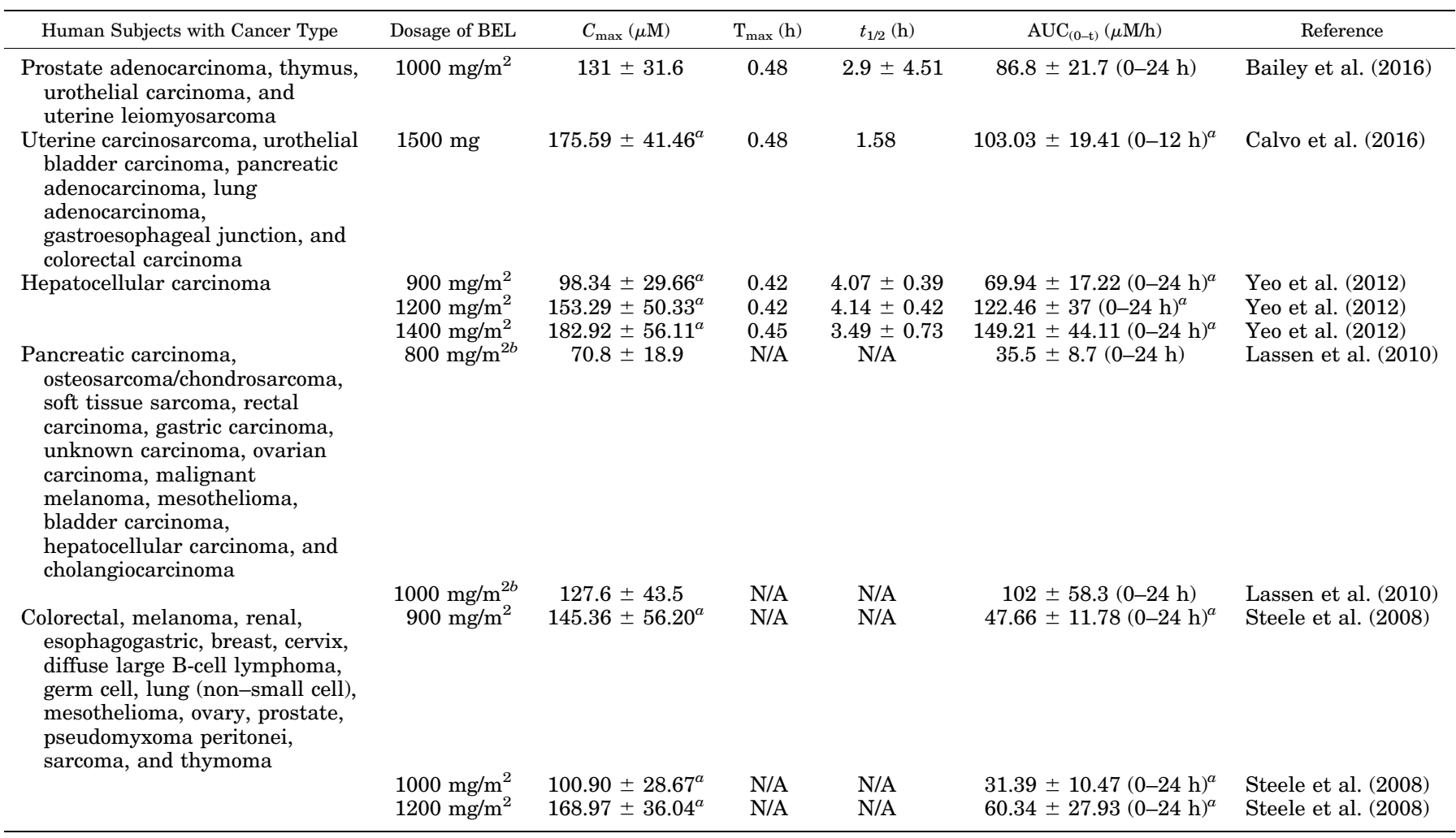

$\mathrm{AUC}_{(0-t)}$, area under the curve for time 0 to present; N/A, not applicable; $\mathrm{T}_{\max }$, time to plasma $C_{\max }$.

${ }^{a}$ Micromolar concentration was calculated based on the provided nanogram per milliliter concentration.

${ }^{b}$ BEL was administered in combination chemotherapy.

expression (Fig. 1B) $(P<0.05)$. Notably, BEL $(1$ or $3 \mu \mathrm{M})$ alone had no effect or a marginally increased CYP3A4 (Fig. 1, A and C) and MDR1 (Fig. 1D) gene expression, suggesting that BEL does not inhibit the basal expression of CYP3A4 and MDR1. Interestingly, BEL failed to abrogate PXR agonist PCN-induced expression of Cyp3a1 in rat primary hepatocytes (Supplemental Fig. 2) $(P<0.05)$. This observation indicates that BEL, at its therapeutic free plasma concentration range in humans, may exhibit species-specific effects on PXR. Together, these results, suggest that BEL can antagonize the agonist-induced hPXR target gene expression at its therapeutically relevant free plasma concentrations.

BEL Is Modestly Cytotoxic at Concentrations Effective for Inhibiting RIF Induction. In CellTiter-Glo Luminescent Cell Viability Assays, $1 \mu \mathrm{M}$ BEL, alone or in combination with RIF, did not exert a noticeable cytotoxicity in the human primary hepatocytes (Fig. 2A) $(P>0.05)$. Although $3 \mu \mathrm{M}$ BEL alone was not cytotoxic, cotreatment of BEL $(3 \mu \mathrm{M})$ with RIF induced a modest cytotoxicity in the hepatocytes (Fig. $2 \mathrm{~A})(P<0.05)$. It is important to note that BEL has been shown to selectively kill human liver cancer cells with an $\mathrm{IC}_{50}$ of approximately $1.5 \mu \mathrm{M}$, without exhibiting significant cytotoxicity in normal hepatocytes (Yeo et al., 2012). This finding is in agreement with the lack of cytotoxic effect of BEL in the human primary hepatocytes at 1 and $3 \mu \mathrm{M}$ in our current study $(P>0.05)$. BEL, however, induced cytotoxicity in the hepatocytes at $10 \mu \mathrm{M}$ (Fig. 2A) $(P<0.05)$. In LS174T human colon cancer cells, BEL exhibited a cytotoxicity only at $10 \mu \mathrm{M}$ when cotreated with RIF (Fig. 2B) $(P<0.05)$. Collectively, these data suggest that BEL can antagonize RIF-induced hPXR target gene expression at its therapeutic free plasma concentrations, with modest cytotoxicity.

BEL Inhibits RIF-Induced CYP3A4 Activity. To determine the BEL inhibition of the hPXR agonist-induced CYP3A4 enzymatic activity, we measured CYP3A4 activity in the human primary hepatocytes using the luminescent P450-Glo CYP3A4 cell-based assays. As described in the Materials and Methods, after 24 hours of treatment with the vehicle or compounds, the media were aspirated, and the hepatocytes were washed with the maintenance media before measuring CYP3A4 activity. This procedure was implemented in an attempt to avoid the direct effects of the compounds or metabolites of the compounds on CYP3A4 activity during the assay. As expected, KET inhibited RIF-induced activity of CYP3A4 $(P<0.05)$ (Fig. 3A). Likewise, BEL inhibited the RIF-induced activity of CYP3A4 $(P<0.05)$ (Fig. 3A), suggesting that BEL antagonizes the hPXR agonist-induced functional expression of CYP3A4.

We did not perform BEL or BEL metabolite distribution studies in the hepatocyte cultures. Therefore, although the culture media were aspirated and the hepatocytes were washed before measuring CYP3A4 activity in the induction studies (Fig. 3A), it is conceivable that some unknown quantities of BEL and/or metabolites of BEL exist inside and outside the hepatocytes during the CYP3A4 assay. However, the fact that BEL did not directly inhibit CYP3A4 activity in the hepatocytes (Fig. 3B) suggests that the measured CYP3A4 activity in the inductions studies (Fig. 3A) would reflect the transcriptional 
A

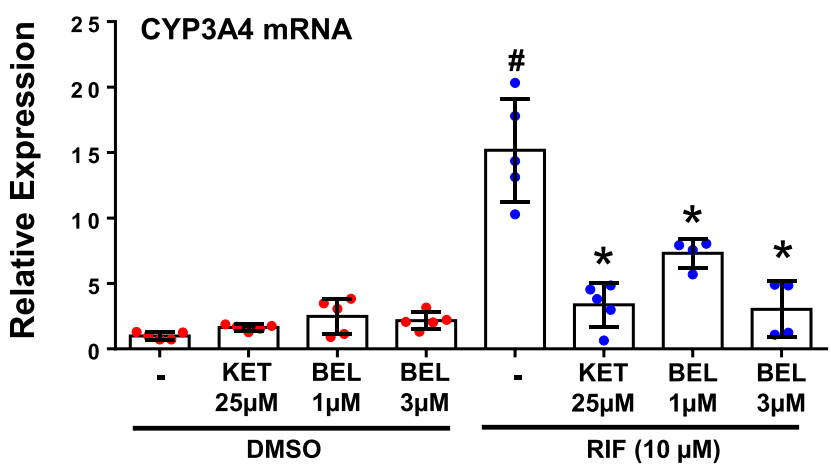

B Human Primary Hepatocytes

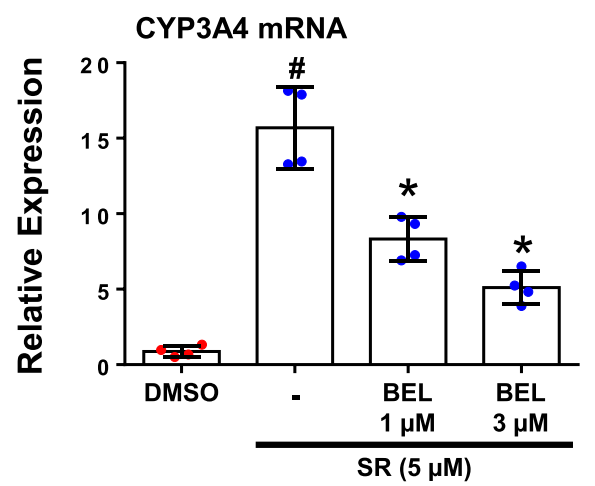

C

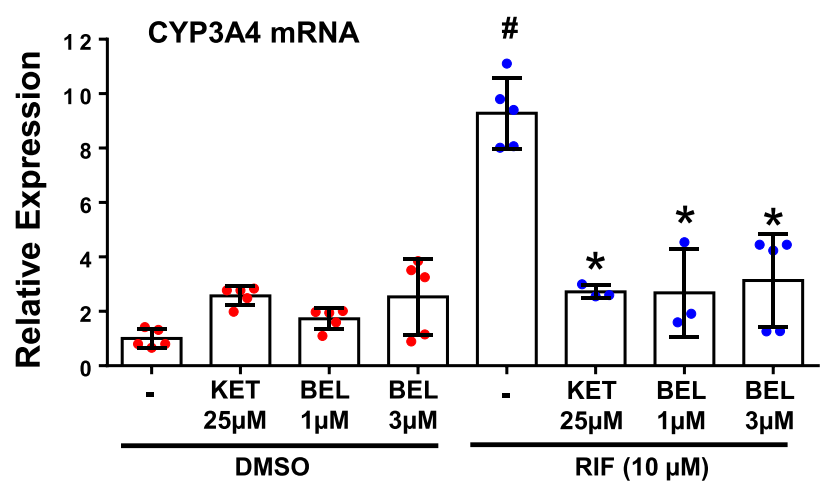

D

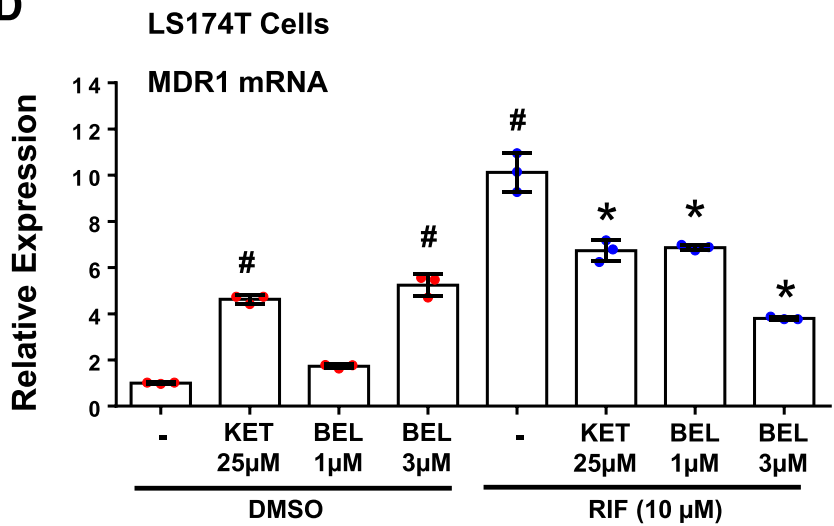

Fig. 1. Effect of BEL on hPXR agonist-induced CYP3A4 and MDR1 gene expression. CYP3A4 and MDR1 mRNA expression was analyzed by quantitative reverse-transcription polymerase chain reaction in human primary hepatocytes (A and B) and LS174T cells (C and D) after treatment with vehicle DMSO, RIF, BEL \pm RIF, SR, BEL + SR, KET, or KET \pm RIF as indicated for 24 hours. Results are presented as the fold change over DMSO treatment. Data are expressed as the mean \pm S.D. values. $P<0.05$, compared with DMSO alone (\#) or RIF or SR alone (*) by ANOVA with Dunnett's multiple-comparisons test.

effects rather than a combination of transcriptional and direct effects of BEL.

BEL Does Not Inhibit the Basal CYP3A4 Activity. Inhibition of the basal CYP3A4 activity, that is, the direct inhibition of CYP3A4 activity, can result in toxicity as a result of reduced metabolism of coadministered CYP3A4 substrates during multidrug therapy. We therefore examined whether BEL inhibits the basal CYP3A4 activity in the cultured human primary hepatocytes. To determine the direct effect of the compounds on CYP3A4 activity, the compounds were added to the hepatocytes only during the assay. KET, a known CYP3A4 inhibitor, diminished the basal activity of CYP3A4 $(P<0.05)$ (Fig. 3B). However, BEL did not affect the basal CYP3A4 activity at the tested $\mathrm{hPXR}$ inhibiting concentrations $(P>0.05)$ (Fig. 3B).

BEL Inhibits RIF-Induced MDR1 Activity. To determine the functional relevance of BEL repression of RIF-induced

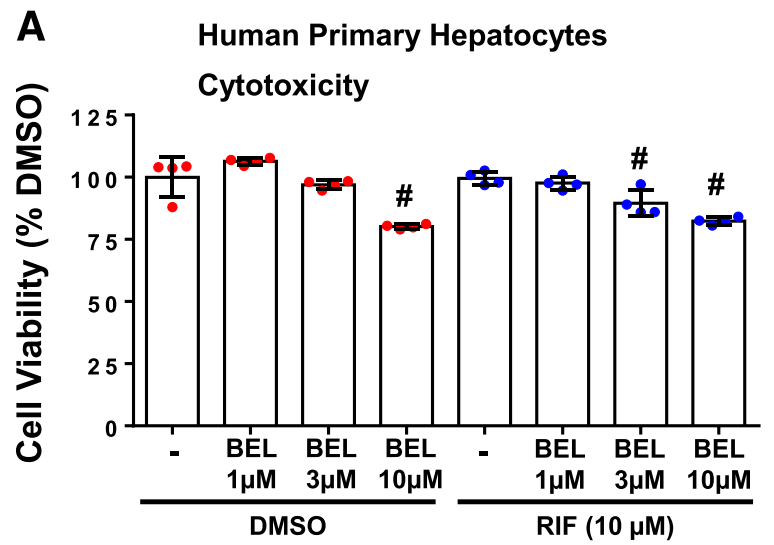

B

LS174T Cells

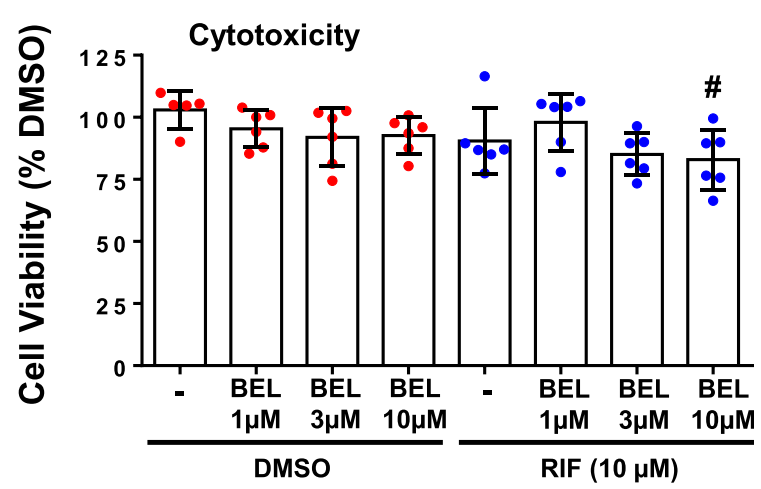

Fig. 2. Effect of BEL on the viability of the hepatocytes and LS174T intestinal cells. Viability of the human primary hepatocytes (A) and LS174T cells (B) was determined under the same experimental conditions indicated in gene expression studies. Cell viability was measured by using CellTiter-Glo Reagent. Viability of DMSO-treated cells was expressed as $100 \%$. Results are shown as the mean \pm S.D. \#P $<0.05$, compared with DMSO alone by ANOVA and Dunnett's multiple-comparisons test. 

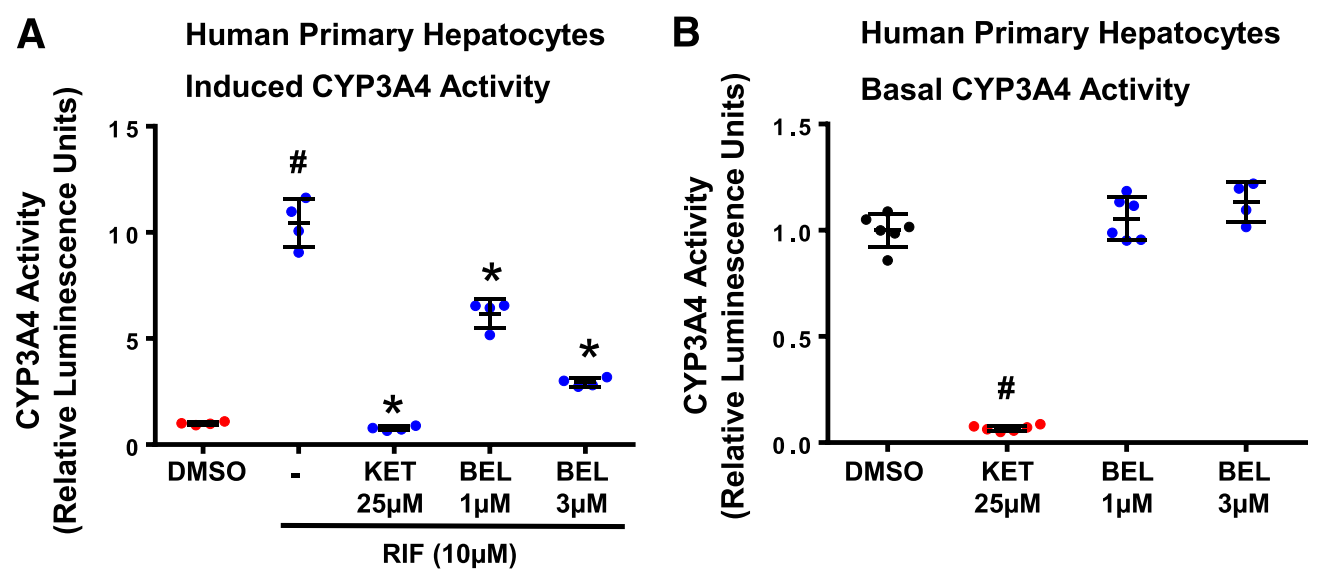

Fig. 3. Effect of BEL on RIF-induced and basal CYP3A4 activity. (A) BEL inhibits RIF-induced CYP3A4 activity in the human primary hepatocytes. CYP3A4 activity was analyzed by the luminescent cytochrome P450-Glo CYP3A4 assays in the human primary hepatocytes after treatment with vehicle, DMSO, RIF, BEL \pm RIF, or KET \pm RIF as indicated for 24 hours. Results are presented as the fold change over DMSO treatment. Data represent the mean \pm S.D. from four independent experiments performed on single-donor hepatocytes. $P<0.05$; compared with DMSO alone (\#) or RIF alone $(*)$ by ANOVA with Dunnett's multiple-comparisons test. (B) BEL does not inhibit the basal CYP3A4 activity in the human primary hepatocytes. DMSO, BEL, or KET was added to the hepatocytes only during the assay period to determine their direct effects on CYP3A4 activity. Results are presented as the fold change over DMSO treatment. Data represent the mean \pm S.D. values from four independent experiments performed on single-donor hepatocytes. \#P $<$ 0.05; compared with DMSO alone by ANOVA with Dunnett's multiple-comparisons test.

MDR1 gene expression, we measured the intracellular accumulation of an MDR1 substrate, R123, in LS174T cells after treatment for 24 hours with DMSO, RIF, BEL, or RIF + BEL (Pondugula et al., 2015a,b). The measurements were performed in the presence or absence of the MDR1-specific inhibitor PSC833. Compared with DMSO, the hPXR agonist RIF decreased R123 accumulation $(P<0.05)$, and this decrease was abolished by the addition of PSC-833 $(P<0.05)$, demonstrating that RIF increases the functional expression of MDR1 (Fig. 4A). Although BEL alone did not affect R123 accumulation $(P>0.05)$, cotreatment of BEL with RIF resulted in increased R123 accumulation $(P<0.05)$ compared with RIF treatment (Fig. 4A), suggesting that BEL attenuates RIF-induced MDR1 functional expression in LS174T cells.

BEL Attenuates RIF-Induced Resistance to SN-38. Previous studies have shown that elevated levels of PXR-mediated expression of drug-metabolizing enzymes and drug-efflux pumps in cancer cells contribute to increased resistance toward chemotherapy drugs (Pondugula and Mani, 2013; Planque et al., 2016; Pondugula et al., 2016). For instance, the activation of hPXR-mediated expression of drug-metabolizing enzymes and drug-efflux pumps decreases the sensitivity of human colon cancer cells, including LS174T cells, to several chemotherapy drugs such as SN-38 [the active metabolite of irinotecan $\left(\mathrm{C}_{22} \mathrm{H}_{20} \mathrm{~N}_{2} \mathrm{O}_{5}\right)$ ] (Pondugula et al., 2016). Because BEL inhibited the hPXR agonist-activated expression of CYP3A4 and MDR1 in LS174 cells, we determined whether BEL inhibits RIF-induced resistance to SN-38. As expected, RIF-induced resistance to $\mathrm{SN}-38$ shown by the decreased sensitivity of LS17T cells to SN-38 after RIF treatment $(P<0.05)$ (Fig. 4B). Notably, BEL increased the sensitivity of LS174T cells to SN-38 by attenuating RIF-induced
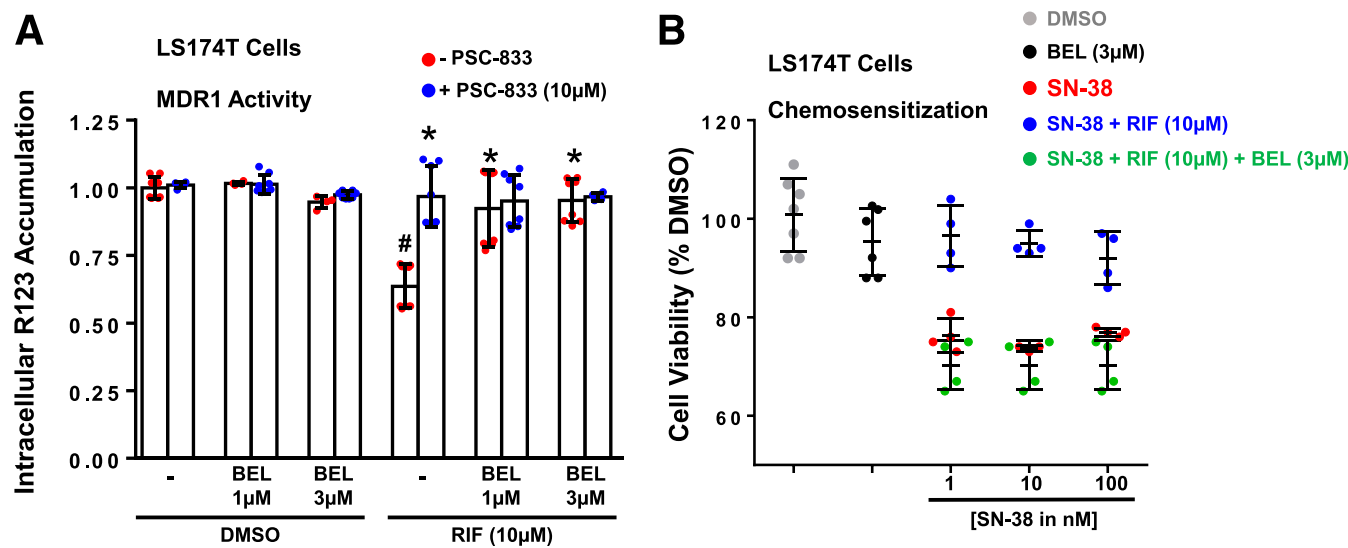

Fig. 4. Effect of BEL on RIF-induced MDR1 activity and RIF-induced resistance to SN-38. (A) BEL inhibits RIF-induced MDR1 activity in LS174T cells. LS174T cells were treated with DMSO, RIF, or BEL \pm RIF as indicated for 24 hours. R123 accumulation was then determined in the absence or presence of the MDR1-specific inhibitor PSC-833. The data are normalized to the DMSO treatment and are presented as the mean \pm S.D. of at least four independent experiments. $P<0.05$, compared with DMSO alone in the absence of PSC-833 (\#) or RIF alone in the absence of PSC-833 (*) by ANOVA and Dunnett's multiple comparisons test. (B) BEL attenuates RIF-induced resistance to SN-38 in LS174T cells. LS174T cells were treated with the indicated compounds for 24 hours, and viability was measured by using the CellTiter-Glo luminescent cell viability assay. The viability of DMSO-treated cells was set to $100 \%$. Data represent the mean \pm S.D. values from at least four experiments. Statistical significance $(P<0.05)$ was determined by ANOVA and Dunnett's multiple-comparisons test. 
resistance to $\mathrm{SN}-38(P<0.05)($ Fig. $4 \mathrm{~B})$. These results suggest that BEL can antagonize hPXR agonist-induced chemoresistance.

Ensemble-Based Molecular Docking Studies Predict High Affinity of BEL for Multiple Sites at hPXR. BEL inhibition of RIF-induced hPXR target gene expression could be attributed to several mechanisms, including BEL interaction with hPXR at multiple sites. The docking score from ensemble-based docking studies predicted that BEL could bind to the LBP, the AF2 region, and the $\alpha 8$ pocket of hPXR (Fig. 5A). BEL shows a relatively high docking score for all the different sites, suggesting its affinity for multiple sites in the hPXR (Fig. 5A). At the LBP, BEL possesses different binding modes (Supplemental Fig. 4), with binding affinity comparable to that of the known LBP-binding compounds like RIF and SR, suggesting that BEL could act as an agonist/antagonist by direct interaction with the LBP. It is interesting to note that the terminal $\mathrm{N}=\mathrm{O}$ group of BEL makes several hydrogen bond interactions with the protein backbone $\mathrm{N}-\mathrm{H}$ or $\mathrm{C}=\mathrm{O}$ groups. These interactions on the one hand impart stability and at the same time can influence the dynamics of hPXR by constraining the backbone movements. A similar scenario was also found when BEL interacts at the $\alpha 8$ site.

Binding at the AF2 site has appealing features, such as the hydrogen bonds with the polar/charged groups (K259 and E427) of hPXR, tying the polar/charged ends of BEL and the hydrophobic residues of the protein and BEL interacting with each other (Fig. 5B). Although these interactions can result in high binding affinity, they need not significantly influence the dynamics of the protein. Notably, the docking score of $\mathrm{BEL}$ is higher for the AF2 region compared with a known PXR antagonist, KET (Fig. 5A), corroborating the weaker affinity of the latter for the AF2 region. Moreover, BEL was found to interact with a number of residues at the AF2 region that are crucial for SRC-1 interaction and effectively mimics the binding of SRC-1 peptide (Fig. 5C). Collectively, the docking studies reveal that BEL has the potential to act as an agonist/ antagonist by binding to the LBP as well as an allosteric antagonist by inhibiting the coactivator binding to the AF2 site. Furthermore, the ensemble-based docking at different sites also identified a stretch of residues shared by the AF2 region and $\alpha 8$ pocket with LBP (Supplemental Fig. 5; Supplemental Table 2). Residues 420-429 and 288-291 were shown to be shared respectively by the AF2 region and the $\alpha 8$ pocket with LBP. Together, these docking results predict that even though BEL has the ability to occupy the LBP, it also has affinity for known sites for allosteric antagonism on hPXR (e.g., AF2 and $\alpha 8$ pocket), thus harnessing potent antagonist properties for BEL.

The docking of BEL to the rat PXR (rPXR) and mouse PXR suggested that BEL could bind to the LBP of PXR from different species, although with varying affinities (Supplemental Table 3). BEL shows a comparatively high docking score for the hPXR and rPXR LBPs than for the mouse PXR. Different binding modes of BEL in the LBPs of rPXR and mouse PXR are depicted in Supplemental Figs. 8 and 9. Similar to hPXR, the terminal $\mathrm{N}=\mathrm{O}$ group of $\mathrm{BEL}$ also makes a hydrogen bond with protein residues, while it interacts with the LBPs of rPXR and mouse PXR. Interestingly, a number of residues conserved across the three different species are found to be involved in the interaction with BEL (Supplemental Fig. 7). The conserved residues Ser247 (Ser244 in rat and mouse), Phe288 (Phe285 in rat and mouse), Trp299 (Trp296 in rat and
A

\begin{tabular}{|llllc|}
\cline { 2 - 5 } Compound & LBP & AF2 & $\alpha 8$ \\
\hline Belinostat & 32.43 & 30.24 & 28.43 \\
Ketoconazole & 32.04 & 14.96 & 16.73 \\
SR12813 & 35.01 & - & - \\
Rifampicin & 31.49 & - & - \\
\hline
\end{tabular}

C

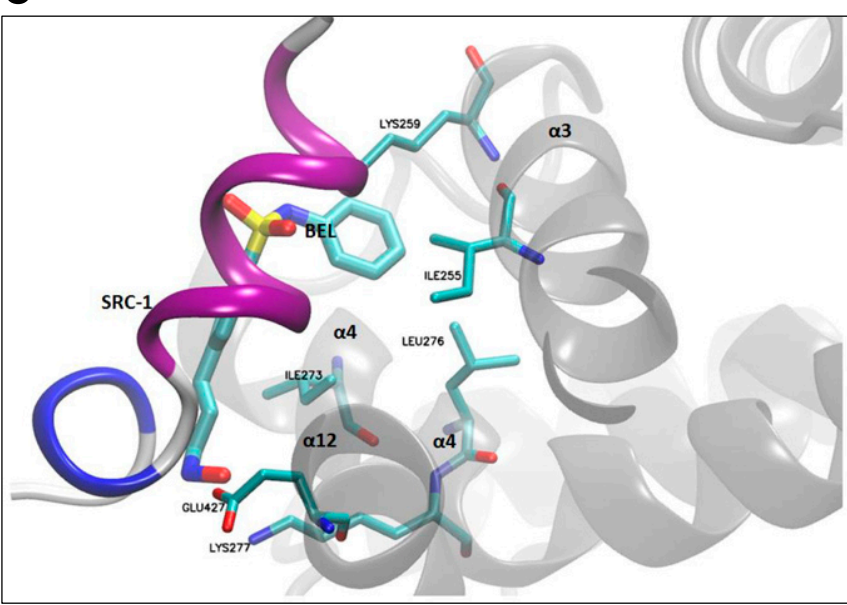

B

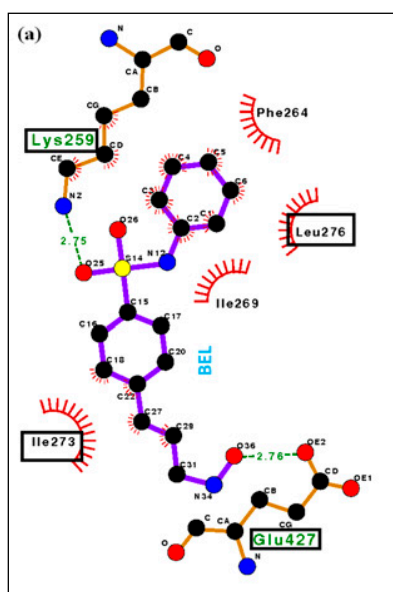

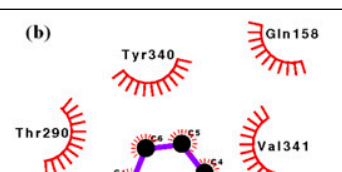

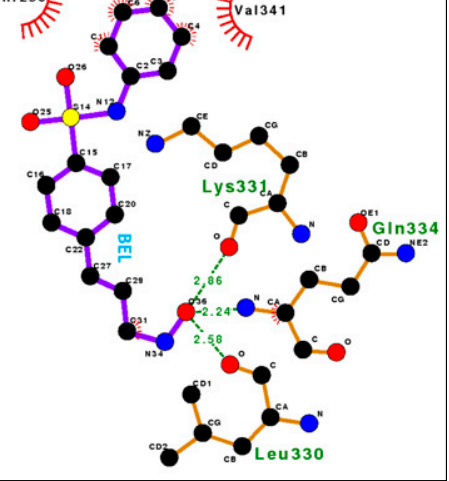

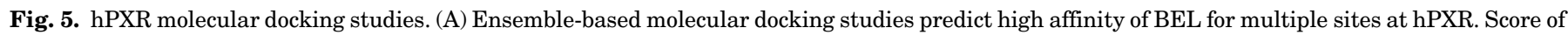

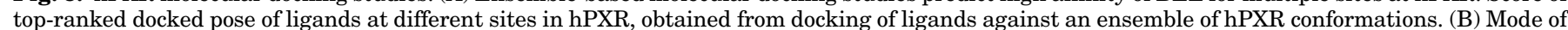

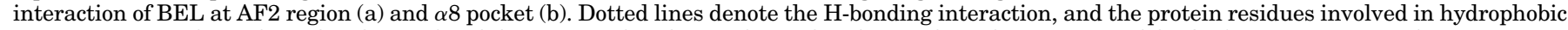

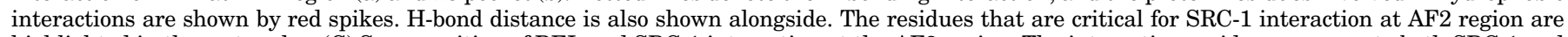

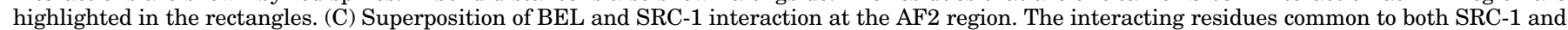
BEL are highlighted. 
mouse), and Met425 (Met422 in rat and mouse) are shown to interact with BEL at the LBP. Additionally, rat and mouse residues corresponding to the interacting residues Asp205, Leu209, Val211, GLn285, Met323, and His407 in humans were also involved in the BEL interaction (Supplemental Fig. 7). Furthermore, it is to be noted that the BEL interacts with Phe305 in the rat LBP (Supplemental Fig. 10), a residue found to be critical for ligand activation (Tirona et al., 2004), whereas the corresponding Leu308 in hPXR fails to network with BEL in the LBP. This finding may explain the differential effect of BEL on hPXR versus rPXR.

BEL Alters SRC-1 Recruitment to hPXR. Coactivators such as SRC-1 contribute to the ligand-induced activation of hPXR. Our docking studies predicted that BEL could interact with some residues at the AF2 region that are important for SRC-1 interaction (Fig. 5C). We therefore tested whether BEL alters the recruitment of SRC-1 to hPXR in a cell-free SRC-1 recruitment assay. SR, a known hPXR agonist, increased SRC-1 recruitment to hPXR $(P<0.05)$ (Fig. 6A). KET, a known PXR inhibitor, decreased the SR-induced interaction of SRC-1 with hPXR $(P<0.05)$ (Fig. 6A). Similar to KET, BEL inhibited SR-induced recruitment of SRC-1 to hPXR $(P<0.05)$ (Fig. 6A). This finding could provide a mechanism for BEL repression of RIF-induced hPXR target genes.

BEL Binds to the Ligand-Biding Domain of hPXR. Our docking studies predicted that BEL could bind to the LBP of hPXR (Fig. 5A). We therefore tested whether BEL binds to the LBD of hPXR in the competitive ligand-binding assays. As expected, SR12813, a known hPXR agonist, bound to the LBD of hPXR (Fig. 6B). BEL bound to the LBD of hPXR but was not as strong as SR (Fig. 6B), suggesting that BEL could act as an agonist/antagonist of $\mathrm{hPXR}$. This result could provide another mechanism for BEL marginal induction of hPXR target genes as well as for BEL repression of RIF-induced hPXR target genes.

\section{Discussion}

Our results show for the first time that BEL, at its clinically relevant therapeutic concentrations, antagonizes the hPXR agonist-induced gene expression and chemoresistance. It is possible to alleviate chemoresistance in some cases if a clinical anticancer drug can antagonize the drug-activated hPXR at therapeutic concentrations during combination chemotherapy. Our results are specifically relevant to cancers where hPXR activation contributes to chemoresistance via the upregulation of hPXR target genes (https://www.oncomine.org) (Pondugula and Mani, 2013; Pondugula et al., 2016).

BEL might be able to alleviate chemoresistance by antagonizing agonist-activated hPXR in tumors. Indeed, in our study, BEL is able to antagonize hPXR agonist-induced chemoresistance in the colon cancer cells (Fig. 4B). Although it remains to be studied, BEL is likely to exhibit such hPXR antagonism in tumors in vivo since BEL concentration in tumor tissues tend to follow that in the plasma. For example, in mice with human ovarian cancer xenografts, after intravenous treatment with a single dose of $200 \mathrm{mg} / \mathrm{kg}$, the BEL concentration in the subcutaneous tumor was found to be similar to that in the plasma or other tissues (Marquard et al., 2008).

The known hPXR antagonists or inhibitors fall short of their clinical use because it is unlikely for these compounds to accomplish in vivo hPXR-inhibiting concentrations without causing unacceptable toxicity (Mani et al., 2013; Chai et al., 2016). For example, with KET, concentrations upward of 10-25 $\mu \mathrm{M}$ would be required to significantly inhibit hPXR function. Although KET is a clinically used drug, it is unlikely that these levels would be achieved safely during the course of treatment. Another major problem with a few hPXR antagonists is that they inhibit the activated hPXR at submicromolar concentrations in in vitro studies (Zhou et al., 2007) but fail to

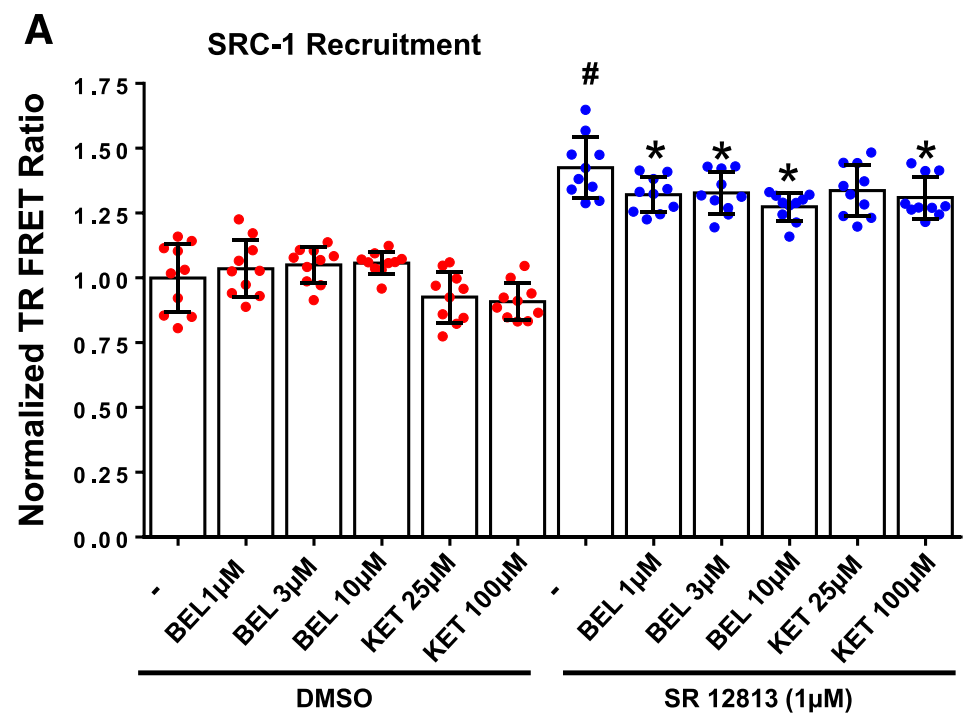

B

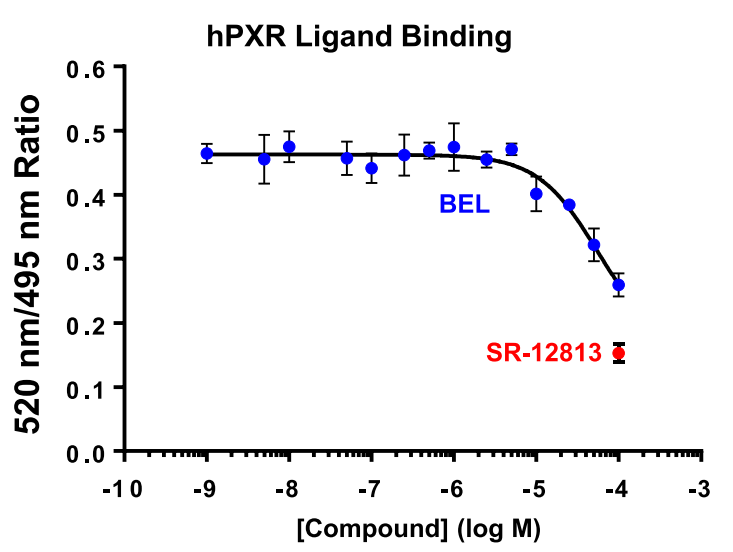

Fig. 6. Mechanisms of BEL interaction with hPXR. (A) BEL affects the recruitment of SRC-1 to the hPXR-LBD in in vitro SRC-1 coactivator recruitment assays. The TR-FRET ratio was calculated by dividing the emission signal at $520 \mathrm{~nm}$ from the acceptor fluorophore by the emission signal at $490 \mathrm{~nm}$ from the donor terbium. The TR-FRET ratios of the compounds were normalized to DMSO. An increase or a decrease of the normalized TR-FRET ratio in the presence of the compounds indicates increased or decreased binding of SRC-1 to hPXR-LBD, respectively. Data represent the mean \pm S.D. values. $P<$ 0.05 , compared with DMSO alone (\#) or SR12813 alone (*) by ANOVA and Dunnett's multiple-comparisons test. (B) BEL binds to hPXR-LBD in an in vitro competitive ligand-binding assay. hPXR-LBD, Tb-anti-GST antibody, and a fluorescein-labeled hPXR ligand tracer were incubated together in the presence of DMSO (vehicle control), BEL (test compound), or SR (a known hPXR agonist). The TR-FRET ratio indicates the binding of the hPXR ligand tracer to hPXR-LBD, and a decrease of the TR-FRET ratio indicates the binding of agonists or antagonists to the hPXR-LBD by outcompeting the binding of the hPXR ligand tracer. Data represent the mean \pm S.D. values from four independent determinations. 
show an in vivo effect in humans. For example, sulforaphane antagonizes hPXR in vitro, but the concentrations needed to sustain this effect were not achieved in vivo, resulting in lack of an hPXR antagonistic effect in in vivo (Poulton et al., 2013). Recently, a novel hPXR antagonist has been discovered (Lin et al., 2017). However, the pharmacokinetics and safety profile of this compound in humans is unknown. BEL is a clinically used anticancer drug with a known pharmacokinetics and safety profile and has the potential to exhibit hPXR antagonism within its therapeutic concentrations.

Adverse drug-drug interactions can occur when therapeutic drugs such as KET directly inhibit the basal activity of CYP3A4. Our results show that BEL, in contrast to KET, does not inhibit the basal CYP3A4 activity (Fig. 3B), suggesting that BEL may not induce CYP3A4-mediated drug-drug interactions during multidrug chemotherapy. Although it was shown in previous in vitro studies (Food and Drug Administration, 2014; Poole, 2014; Lee et al., 2015) that BEL and the metabolites of BEL can inhibit CYP2C8 and CYP2C9, there was no indication of whether BEL can inhibit CYP3A4.

During drug therapy, although elevated levels of hPXRregulated drug-metabolizing enzymes and drug-efflux pumps can compromise drug efficacy, reduced basal levels of the same hPXR target genes can lead to drug toxicity. Our results show that BEL does not inhibit the basal expression or activity of the hPXR target genes, while downregulating the hPXR agonistinduced gene expression and activity. These findings suggest that BEL has the potential to enhance drug efficacy by inhibiting drug-activated hPXR without causing drug toxicity during multidrug chemotherapy. Indeed, BEL attenuates RIF-induced resistance to the chemotherapy drug SN-38, suggesting that BEL may enhance the efficacy of chemotherapy drugs by inhibiting the drug-activated hPXR (Fig. 4B).

BEL Inhibition of RIF-Induced hPXR Target Inhibition Could Be Due to Multiple Mechanisms. Our molecular docking studies predict that BEL could directly interact with $\mathrm{hPXR}$ via the LBP, the $\alpha 8$ pocket, and the AF2 regions of hPXR. Indeed, BEL binds to the LBD of hPXR (Fig. 6B) and attenuates the agonist-increased interaction of SRC-1 with hPXR (Fig. 6A). It is also possible that BEL inhibits the agonist-activated hPXR by binding to the $\alpha 8$ pocket of hPXR, although it remains to be experimentally determined. Collectively, these results suggest that BEL has the potential to act as an antagonist by binding to the LBP as well as to the AF2 site and possibly to the $\alpha 8$ pocket.

Recent work (Tonddast-Navaei et al., 2017) has shown the importance of multiple ligands binding to multiple sites as well as to the same site. Multiple sites either can be totally independent or can be formed of shared regions. It is interesting to see that the LBP of hPXR shares some regions with AF2 as well as with $\alpha 8$ (Supplemental Fig. 5; Supplemental Table 2). Such a shared region can mutually influence the binding and activity of ligands at multiple sites. Ensemble-based docking identified protein conformations where BEL can coexist in multiple sites in the hPXR at the same time (Supplemental Fig. 6), where BEL maintains its typical nature of interaction. Additionally, recent studies (Lin et al., 2017) have shown that the ligand interaction with residues of helix $\alpha 12$, a shared region between the LBP and the AF2 region (Supplemental Fig. 5; residues 420-429), is found to be critical for determining the agonist/antagonist activity of a compound.
BEL exhibited differential effects on the exogenous hPXR reporter gene expression when compared with the endogenous hPXR target gene expression. In LS180 cells, although BEL alone showed no effect on the hPXR transactivation function (Supplemental Fig. 11A) $(P>0.05)$, it inhibited RIF-induced hPXR transactivation of the reporter gene (Supplemental Fig. 11B) $(P<0.05)$. However, in human embryonic kidney-293 cells, BEL not only induced hPXR transactivation of the reporter gene (Supplemental Fig. 11C) $(P<0.05)$, but also potentiated RIF-induced hPXR transactivation (Supplemental Fig. 11D) $(P<0.05)$. Similarly, BEL showed the agonistic (Supplemental Fig. 11E) $(P<0.05)$ and potentiation effects (Supplemental Fig. 11F) $(P<0.05)$ in LS174T cells. In parallel cell viability assays, BEL exhibited moderate cytotoxicity in LS180 cells (Supplemental Fig. 12A) $(P<0.05)$ and human embryonic kidney-293 cells (Supplemental Fig. 12B) $(P<0.05)$, with no cytotoxic effect in LS174T cells (Supplemental Fig. 12C) $(P>0.05)$.

Although BEL showed consistent effects on the endogenous hPXR target gene expression in both hepatocytes and intestinal cells, it displayed differential effects on the exogenous gene expression, depending on the cell line. It is known that the regulation of PXR-dependent reporter gene activity using transfected cells does not always correlate with the regulation of endogenous hPXR target gene expression (Luo et al., 2002; Cui et al., 2008). It is also known that PXR activity can be regulated in a tissue-specific (Robbins and Chen, 2014) and contextdependent (Pondugula et al., 2016) manner with in the same cells. Our reporter gene results show a differential effect of BEL on PXR in a cell-dependent manner. The observed discrepancies in our studies between the endogenous and overexpression systems could be attributed to an imbalance of PXR regulation machinery in the overexpression systems.

It is possible that the observed effects of BEL on the endogenous gene expression in the hepatocytes and intestinal cells could have also been contributed by BEL metabolites. Future studies are warranted to investigate the effect of BEL metabolites on the PXR target gene expression. Future studies are also required to examine whether BEL is selective toward hPXR. More importantly, it remains to be studied whether BEL inhibits hPXR agonist-induced gene expression and chemoresistance in vivo.

In conclusion, our results show that BEL, at its clinically therapeutic concentration, can antagonize agonist-activated hPXR target gene expression and chemoresistance. Thus, BEL could be a potential candidate to overcome hPXR agonistinduced chemoresistance during combination chemotherapy in some cancer cases (Fig. 7).

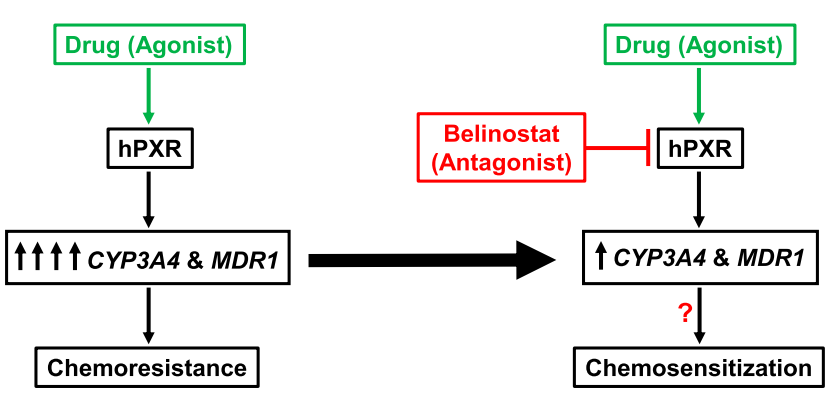

Fig. 7. Proposed model for BEL repression of hPXR agonist-induced target gene expression and chemoresistance during combination chemotherapy. 


\section{Acknowledgments}

We thank Dr. Coleman, Dr. Mansour, Dr. Schwartz, Dr. Irwin, Dr. Biancardi, and Dr. Tao for sharing their research facilities. The authors would also like to give special acknowledgment to Angel Potterfield-Pritchett for her support throughout the entirety of this project.

\section{Authorship Contributions}

Participated in research design: Pondugula.

Conducted experiments: Abbott, Chaudhury, Dvorak, Jiskrova,

Poulikova, Vyhlidalova, Pondugula

Contributed new reagents or analytic tools: Chandran, Vishveshwara, Mani.

Performed data analysis: Abbott, Chandran, Vishveshwara,

Dvorak, Jiskrova, Poulikova, Vyhlidalova, Mani, Pondugula.

Wrote or contributed to the writing of the manuscript: Abbott, Chandran, Vishveshwara, Dvorak, Mani, Pondugula.

\section{References}

Bailey H, McPherson JP, Bailey EB, Werner TL, Gupta S, Batten J, Reddy G, Bhat G, Sharma S, and Agarwal N (2016) A phase I study to determin the pharmacokinetics and urinary excretion of belinostat and metabolites in patients with advanced solid tumors. Cancer Chemother Pharmacol $\mathbf{7 8}$ 1059-1071.

Banerjee M, Robbins D, and Chen T (2013) Modulation of xenobiotic receptors by steroids. Molecules 18:7389-7406.

Borrelli F and Izzo AA (2009) Herb-drug interactions with St John's wort (Hypericum perforatum): an update on clinical observations. AAPS J 11:710-727.

Calvo E, Reddy G, Boni V, García-Cañamaque L, Song T, Tjornelund J, Choi MR, and Allen LF (2016) Pharmacokinetics, metabolism, and excretion of (14)C-labeled belinostat in patients with recurrent or progressive malignancies. Invest New Drugs 34:193-201.

Chai SC, Cherian MT, Wang YM, and Chen T (2016) Small-molecule modulators of PXR and CAR. Biochim Biophys Acta 1859:1141-1154.

Chandran A and Vishveshwara S (2016) Exploration of the conformational landscape in pregnane X receptor reveals a new binding pocket. Protein Sci $\mathbf{2 5}$ : 1989-2005.

Chang TK and Waxman DJ (2006) Synthetic drugs and natural products as modulators of constitutive androstane receptor (CAR) and pregnane $\mathrm{X}$ receptor (PXR). Drug Metab Rev 38:51-73.

Chen Y, Tang Y, Guo C, Wang J, Boral D, and Nie D (2012) Nuclear receptors in the multidrug resistance through the regulation of drug-metabolizing enzymes and drug transporters. Biochem Pharmacol 83:1112-1126.

Choo EF, Woolsey S, DeMent K, Ly J, Messick K, Qin A, and Takahashi R (2015) Use of transgenic mouse models to understand the oral disposition and drug-drug interaction potential of cobimetinib, a MEK inhibitor. Drug Metab Dispos 43:864-869.

Cui X, Thomas A, Gerlach V, White RE, Morrison RA, and Cheng KC (2008) Application and interpretation of $\mathrm{hPXR}$ screening data: validation of reporter signal requirements for prediction of clinically relevant CYP3A4 inducers. Biochem Pharmacol 76:680-689.

Dvorak Z and Pavek P (2010) Regulation of drug-metabolizing cytochrome P450 enzymes by glucocorticoids. Drug Metab Rev 42:621-635.

El-Sankary W, Plant NJ, Gibson GG, and Moore DJ (2000) Regulation of the CYP3A4 gene by hydrocortisone and xenobiotics: role of the glucocorticoid and pregnane $\mathrm{X}$ receptors. Drug Metab Dispos 28:493-496.

Food and Drug Administration (2014) Beleodaq (belinostat) for injection, in Approved Drug Products with Therapeutic Equivalence Evaluations. Food and Drug Administration, White Oak, MD.

Hu ZP, Yang XX, Chen X, Cao J, Chan E, Duan W, Huang M, Yu XQ, Wen JY, and Zhou SF (2007) A mechanistic study on altered pharmacokinetics of irinotecan by St. John's wort. Curr Drug Metab 8:157-171.

Huang H, Wang H, Sinz M, Zoeckler M, Staudinger J, Redinbo MR, Teotico DG, Locker J, Kalpana GV, and Mani S (2007) Inhibition of drug metabolism by blocking the activation of nuclear receptors by ketoconazole. Oncogene 26:258-268

Jones G, Willett P, Glen RC, Leach AR, and Taylor R (1997) Development and validation of a genetic algorithm for flexible docking. J Mol Biol 267: $727-748$.

Laribi K, Alani M, Truong C, and Baugier de Materre A (2018) Recent advances in the treatment of peripheral T-cell lymphoma. Oncologist 23:1039-1053.

Lassen U, Molife LR, Sorensen M, Engelholm SA, Vidal L, Sinha R, Penson RT, Buhl-Jensen P, Crowley E, Tjornelund J, et al. (2010) A phase I study of the safety and pharmacokinetics of the histone deacetylase inhibitor belinostat administered in combination with carboplatin and/or paclitaxel in patients with solid tumours. $\mathrm{Br}$ J Cancer 103:12-17.

Lee CM, Kumar V, Riley RI, and Morgan ET (2010) Metabolism and action of proteasome inhibitors in primary human hepatocytes. Drug Metab Dispos 38: $2166-2172$.

Lee HZ, Kwitkowski VE, Del Valle PL, Ricci MS, Saber H, Habtemariam BA, Bullock J, Bloomquist E, Li Shen Y, Chen XH, et al. (2015) FDA approval: belinostat for the treatment of patients with relapsed or refractory peripheral T-cell lymphoma. Clin Cancer Res 21:2666-2670.
Lin KT and Wang LH (2016) New dimension of glucocorticoids in cancer treatment. Steroids 111:84-88.

Lin W, Wang YM, Chai SC, Lv L, Zheng J, Wu J, Zhang Q, Wang YD, Griffin PR and Chen T (2017) SPA70 is a potent antagonist of human pregnane $\mathrm{X}$ receptor. Nat Commun 8:741.

Luo G, Cunningham M, Kim S, Burn T, Lin J, Sinz M, Hamilton G, Rizzo C, Jolley S, Gilbert D, et al. (2002) CYP3A4 induction by drugs: correlation between a pregnane $\mathrm{X}$ receptor reporter gene assay and CYP3A4 expression in human hepatocytes. Drug Metab Dispos 30:795-804.

MacLeod AK, McLaughlin LA, Henderson CJ, and Wolf CR (2015) Activation status of the pregnane $\mathrm{X}$ receptor influences vemurafenib availability in humanized mouse models. Cancer Res 75:4573-4581.

Mani S, Dou W, and Redinbo MR (2013) PXR antagonists and implication in drug metabolism. Drug Metab Rev 45:60-72.

Marquard L, Petersen KD, Persson M, Hoff KD, Jensen PB, and Sehested M (2008) Monitoring the effect of belinostat in solid tumors by $\mathrm{H} 4$ acetylation. APMIS 116:382-392.

Masuyama H, Suwaki N, Tateishi Y, Nakatsukasa H, Segawa T, and Hiramatsu Y (2005) The pregnane $\mathrm{X}$ receptor regulates gene expression in a ligand- and promoter-selective fashion. Mol Endocrinol 19:1170-1180.

Nguyen L, Holland J, Miles D, Engel C, Benrimoh N, O'Reilly T, and Lacy S (2015) Pharmacokinetic (PK) drug interaction studies of cabozantinib: effect of CYP3A inducer rifampin and inhibitor ketoconazole on cabozantinib plasma $\mathrm{PK}$ and effect of cabozantinib on CYP2C8 probe substrate rosiglitazone plasma PK. J Clin Pharmacol 55:1012-1023.

Pascussi JM, Drocourt L, Fabre JM, Maurel P, and Vilarem MJ (2000) Dexamethasone induces pregnane $\mathrm{X}$ receptor and retinoid $\mathrm{X}$ receptor-alpha expression in human hepatocytes: synergistic increase of CYP3A4 induction by pregnane X receptor activators. Mol Pharmacol 58:361-372.

Pascussi JM, Drocourt L, Gerbal-Chaloin S, Fabre JM, Maurel P, and Vilarem MJ (2001) Dual effect of dexamethasone on CYP3A4 gene expression in human hepatocytes. Sequential role of glucocorticoid receptor and pregnane X receptor. Eur $J$ Biochem 268:6346-6358.

Piper WL, Waddell JA, and Solimando DA Jr (2014) Drug monographs: belinostat and idelalisib. Hosp Pharm 49:1009-1013.

Planque C, Rajabi F, Grillet F, Finetti P, Bertucci F, Gironella M, Lozano JJ, Beucher B, Giraud J, Garambois V, et al. (2016) Pregnane X-receptor promotes stem cellmediated colon cancer relapse. Oncotarget 7:56558-56573.

Pondugula SR, Ferniany G, Ashraf F, Abbott KL, Smith BF, Coleman ES, Mansour M, Bird RC, Smith AN, Karthikeyan C, et al. (2015a) Stearidonic acid, a plantbased dietary fatty acid, enhances the chemosensitivity of canine lymphoid tumor cells. Biochem Biophys Res Commun 460:1002-1007.

Pondugula SR, Flannery PC, Abbott KL, Coleman ES, Mani S, Samuel T, and Xie W (2015b) Diindolylmethane, a naturally occurring compound, induces CYP3A4 and MDR1 gene expression by activating human PXR. Toxico Lett 232:580-589.

Pondugula SR, Flannery PC, Apte U, Babu JR, Geetha T, Rege SD, Chen T, and Abbott KL (2015c) Mg2+/Mn2+-dependent phosphatase $1 \mathrm{~A}$ is involved in regulating pregnane $\mathrm{X}$ receptor-mediated cytochrome p450 3A4 gene expression. Drug Metab Dispos 43:385-391.

Pondugula SR and Mani S (2013) Pregnane xenobiotic receptor in cancer pathogenesis and therapeutic response. Cancer Lett 328:1-9.

Pondugula SR, Pavek P, and Mani S (2016) Pregnane X receptor and cancer: contextspecificity is key. Nucl Receptor Res 3:101198.

Poole RM (2014) Belinostat: first global approval. Drugs 74:1543-1554.

Poulton EJ, Levy L, Lampe JW, Shen DD, Tracy J, Shuhart MC, Thummel KE, and Eaton DL (2013) Sulforaphane is not an effective antagonist of the human pregnane X-receptor in vivo. Toxicol Appl Pharmacol 266:122-131.

Robbins D and Chen T (2014) Tissue-specific regulation of pregnane X receptor in cancer development and therapy. Cell Biosci 4:17.

Schuetz E, Lan L, Yasuda K, Kim R, Kocarek TA, Schuetz J, and Strom S (2002) Development of a real-time in vivo transcription assay: application reveals pregnane X receptor-mediated induction of CYP3A4 by cancer chemotherapeutic agents. Mol Pharmacol 62:439-445.

Steele NL, Plumb JA, Vidal L, Tjørnelund J, Knoblauch P, Rasmussen A, Ooi CE Buhl-Jensen P, Brown R, Evans TR, et al. (2008) A phase 1 pharmacokinetic and pharmacodynamic study of the histone deacetylase inhibitor belinostat in patients with advanced solid tumors. Clin Cancer Res 14:804-810.

Synold TW, Dussault I, and Forman BM (2001) The orphan nuclear receptor SXR coordinately regulates drug metabolism and efflux. Nat Med 7:584-590.

Takeshita A, Igarashi-Migitaka J, Nishiyama K, Takahashi H, Takeuchi Y, and Koibuchi N (2011) Acetyl tributyl citrate, the most widely used phthalate substitute plasticizer, induces cytochrome p450 3a through steroid and xenobiotic receptor. Toxicol Sci 123:460-470.

Tirona RG, Leake BF, Podust LM, and Kim RB (2004) Identification of amino acids in rat pregnane $\mathrm{X}$ receptor that determine species-specific activation. Mol Pharmacol 65:36-44.

Tonddast-Navaei S, Srinivasan B, and Skolnick J (2017) On the importance of composite protein multiple ligand interactions in protein pockets. $J$ Comput Chem 38:1252-1259.

Veith H, Southall N, Huang R, James T, Fayne D, Artemenko N, Shen M, Inglese J, Austin CP, Lloyd DG, et al. (2009) Comprehensive characterization of cytochrome P450 isozyme selectivity across chemical libraries. Nat Biotechnol 27:1050-1055

Wang H, Faucette SR, Gilbert D, Jolley SL, Sueyoshi T, Negishi M, and LeCluyse EL (2003) Glucocorticoid receptor enhancement of pregnane X receptor-mediated CYP2B6 regulation in primary human hepatocytes. Drug Metab Dispos 31:620-630.

Xiao L, Nickbarg E, Wang W, Thomas A, Ziebell M, Prosise WW, Lesburg CA, Taremi SS, Gerlach VL, Le HV, et al. (2011) Evaluation of in vitro PXR-based assays and 
in silico modeling approaches for understanding the binding of a structurally diverse set of drugs to PXR. Biochem Pharmacol 81:669-679.

Yeo W, Chung HC, Chan SL, Wang LZ, Lim R, Picus J, Boyer M, Mo FK, Koh J Rha SY, et al. (2012) Epigenetic therapy using belinostat for patients with unresectable hepatocellular carcinoma: a multicenter phase I/II study with biomarker and pharmacokinetic analysis of tumors from patients in the Mayo Phase II Consortium and the Cancer Therapeutics Research Group. J Clin Oncol 30:3361-3367.

Zhou C, Poulton EJ, Grün F, Bammler TK, Blumberg B, Thummel KE, and Eaton DL (2007) The dietary isothiocyanate sulforaphane is an antag onist of the human steroid and xenobiotic nuclear receptor. Mol Pharmacol 71:220-229.
Zuo R, Li F, Parikh S, Cao L, Cooper KL, Hong Y, Liu J, Faris RA, Li D, and Wang H (2017) Evaluation of a novel renewable hepatic cell model for prediction of clinical CYP3A4 induction using a correlation-based relative induction score approach. Drug Metab Dispos 45:198-207.

Address correspondence to: Satyanarayana R. Pondugula, Department of Anatomy, Physiology and Pharmacology, College of Veterinary Medicine, Auburn University, 109 Greene Hall, Auburn, AL 36849. E-mail: srp0010@ auburn.edu; or Sridhar Mani, Albert Einstein Cancer Center, Albert Einstein College of Medicine, New York, NY. E-mail: sridhar.mani@einstein.yu.edu 